\title{
Transepithelial Laser versus Alcohol Assisted Photorefractive Keratectomy Safety and Efficacy: 1-Year Follow-up of a Contralateral Eye Study
}

\author{
Hesham Mohamed Gharieb, ${ }^{1,2}$, Mo'mena Ahmad A Awad-Allah ${ }^{1}$, Anas Adel Ahmed²,3 Ihab Saad Othman²,4 \\ ${ }^{1}$ Department of Ophthalmology, Faculty of Medicine, Ain Shams University, Cairo, Egypt \\ ${ }^{2}$ Eye World Hospital, Dokki, Giza, Egypt \\ ${ }^{3}$ Department of Ophthalmology, Faculty of Medicine, Helwan University, Cairo, Egypt \\ ${ }^{4}$ Department of Ophthalmology, Faculty of Medicine, Cairo University, Giza, Egypt
}

Purpose: To compare single-step transepithelial photorefractive keratectomy (TPRK) to conventional alcohol assisted epithelial removal then photorefractive keratectomy (AAPRK) regarding pain, epithelial healing, visual acuity, corneal haze measured subjectively and objectively, higher order aberrations changes, contrast sensitivity and vector analysis of astigmatic correction with one year follow-up.

Methods: A prospective double-blind randomized study of 29 subjects (58 eyes) who underwent myopic aberration-free laser correction by smart pulse technology using Schwind Amaris $1050 \mathrm{~Hz}$ with 1-year follow-up. Right eye was randomly treated by AAPRK or TPRK. Postoperative assessment was performed on day 1 and 3, at 1st week, and 1st, 3rd, 6th, and 12th months. Patients were assessed for pain, epithelial healing, visual acuity, corneal haze, astigmatic correction, higher order aberrations and contrast sensitivity.

Results: Epithelial healing was complete by the 3rd day in $62.1 \%$ of AAPRK eyes and in $89.7 \%$ of TPRK eyes. First day postoperative pain was higher in TPRK group $(p=0.0134)$. The decimal uncorrected visual acuity at 12 months was $1.47 \pm 0.39$ and $1.57 \pm 0.38$ in the AAPRK and TPRK groups respectively $(p=0.3719)$. Post-photorefractive keratectomy haze reached a final level of $0.04 \pm 0.14$ and $0.02 \pm 0.1$ in AAPRK and TPRK groups respectively ( $p=0.5607$ ). Contrast sensitivity was comparable in low and high frequency cycles per degree. Vector analysis of astigmatic correction showed correction index at one year of 0.99 and 1.05 for AAPRK and TPRK groups respectively.

Conclusions: Alcohol assisted and transepithelial photorefractive keratectomy have comparable results regarding safety and efficacy.

Key Words: Alcohol assisted photorefractive keratectomy, Contrast sensitivity, Smart pulse technology, Transepithelial photorefractive keratectomy, Vector analysis

Received: August 14, 2020 Final revision: October 17, 2020

Accepted: January 6, 2021

Corresponding Author: Mo'mena Ahmad A Awad-Allah, MD, FRCSGlasgow. Department of Ophthalmology, Faculty of Medicine, Ain Shams University, 83rd Othman Buildings, Ali Ameen Street, Nasr City, Cairo 11727, Egypt. Tel: 20-10-1515-9486, Fax: 20-2-2434-6753, E-mail: momena@med.asu.edu.eg
Photorefractive keratectomy (PRK) was described for the first time by Munnerlyn et al. [1] in their paper published in 1988. The original technique depends on mechanical removal of the corneal epithelium, and later alcohol use was tried [2]. Transepithelial PRK (TPRK) was described in the late 1990s by Clinch et al. [3] as an alterna- 
tive to the conventional mechanical or alcohol assisted removal of the epithelium. The advantages of TPRK include; no instrumental direct contact with the eye, reduced procedure time and the potential to minimize the epithelial raw area needed for the correcting ablation [4]. Avoiding using alcohol is alleged to avoid the potential toxicity to the limbal stem cells, decrease the postoperative pain and corneal haze with rapid healing time and a faster visual recovery $[5,6]$.

The new Schwind Amaris $1050 \mathrm{~Hz}$ excimer laser (Schwind Eye-Tech-Solutions GmbH, Kleinostheim, Germany) have developed smart pulse technology (SPT) which aimed to minimize residual stromal bed surface irregularities at end of treatment. SPT laser spots have a truncated super-Gaussian spot shape. This profile is flatter than Gaussian but rounder than flat-top profiles. The laser spot peak intensity was truncated by $14 \%$ to remove the subthreshold laser spot flanks, this decreases thermal load and ablation effect of the lateral laser pulse energy not utilized in true ablation of the bed $[7,8]$. Combining SPT with transepithelial laser ablation; the Amaris $1050 \mathrm{~Hz}$ uses SmartSurface technology (Schwind Eye-Tech-Solutions GmbH) for TPRK treatment. It has the ability to adjust to the personal and individual variance in epithelial thickness due to different epithelial and stromal profiles. The surgeon performs surface ablation using a nomogram that combines epithelial removal with spherocylindrical correction through delivering a varying quantity of laser energy initiated at the center to the periphery [9].

This study aimed at comparing single-step TPRK to conventional alcohol assisted epithelial removal regarding pain, epithelial healing, visual acuity, corneal haze measured subjectively and objectively, astigmatic correction, higher order aberrations, contrast sensitivity and vector analysis of astigmatic correction with one year follow-up.

\section{Materials and Methods}

\section{Patients}

This study was a prospective double-blind randomized study. According to sample size calculation, 29 subjects (58 eyes) were included. One patient had postoperative papilledema due to idiopathic increase intracranial pressure and one with irregular follow-up. Both were excluded from statistical analysis with final number of 54 eyes at the end of the 12 months. This was within the precalculated dropout rate $(10 \%)$. Technique of PRK (either TPRK or alcohol assisted PRK [AAPRK]) used for right eye was chosen randomly by online random number generator. This study design was chosen based on the need for a matched control group to eliminate individual variations potentially affecting the outcome.

The study was approved by Cairo University Ethical Committee (17220215). The study also followed the tenets of the Declaration of Helsinki. Both procedures were explained in simple words for all patients, then, a written informed consent was obtained prior to the surgery. The patients enrolled in this study underwent surgery at Eye World Hospital in Giza, Egypt from September 2018 to February 2019 and completed one-year follow-up by February 2020 . They were all done by a single experienced surgeon (HG). The inclusion criteria were age over 18 years, myopic refraction with or without astigmatism (not exceeding 3 diopters [D]) with a spherical equivalent between -1.5 and $-7 \mathrm{D}$, a stable refraction for 18 months prior to the surgery, a maximum planned corneal stromal ablation (without epithelium) of $90 \mu \mathrm{m}$, a preoperative-higher order aberrations $<0.50$ in both eyes and a corrected Snellen's distance visual acuity of 0.63 decimal or better. Exclusion criteria were presence of corneal epithelial pathology (dystrophies or scarring), keratoconus, eyelids or conjunctival infections, glaucoma, cataract, posterior segment pathology, previous eye surgeries, or positive systemic medical history. All patients received bilateral treatment by the same surgeon at the same session.

\section{Preoperative assessment}

Patients were instructed to stop using soft contact lenses 3 weeks prior to examination for surgery. The preoperative assessment included uncorrected visual acuity (UCVA) and best-corrected visual acuity using Snellen's charts. Manifest and cycloplegic refraction were measured with the Potec PRK-7000 autorefractor/keratometer (Potec Co., Daejeon, Korea). Intraocular pressure measurement was done by the Pulsair Desktop Tonometer (Keeler, Windsor, United Kingdom). Clinical examination with the slit-lamp biomicroscopy, including dilated fundus assessment of the posterior segment was done to all patients. Patients' corneas were assessed with Pentacam HR (Oculus Optikger- 
ate $\mathrm{GmbH}$, Wetzlar, Germany) with software ver. 1.21r.65 and with the Combi Wavefront Analyzer including both functions of the Schwind Sirius (Schwind Eye-Tech-Solutions) topography imaging and the ocular aberrometer function of the Sirius device (CSO ver. 3.2.1.60; Costruzione Strumenti Oftalmici, Florence, Italy).

\section{Surgical technique}

In both eyes, surgical preparation with topical anesthetic and povidone iodine $5 \%$ instillation, prior to draping and insertion of a speculum were done. In the conventional (alcohol assisted) PRK eyes, treatment was with the standard PRK protocol. Epithelial delamination was performed with $20 \%$ ethanol applied using a $9 \mathrm{~mm}$ well for 10 seconds, followed by epithelial removal with a spatula. This step avoided uneven wetting and the subsequent risk of uneven ablation. In the transepithelial eyes, the excimer laser administration was in a single continuous session to ablate both the epithelium and stroma in a single step. According to a population-based epithelium-thickness profile, the ablation plan utilized $55 \mu \mathrm{m}$ centrally, and $65 \mu \mathrm{m}$ peripherally with an $8 \mathrm{~mm}$ ablation zone, in which an even laser energy on the entire corneal surface was provided.

Both eye groups treatment was with the Amaris $1050 \mathrm{~Hz}$ excimer laser with SPT and SmartSurface treatment in the TPRK group. Both groups ablation profiles were aspheric aberration neutral (non-wavefront guided treatment). Eye movements throughout the ablation were compensated by static and dynamic cyclotorsion corrections. All eyes in both groups received a standardized intraoperative application of mitomycin-C $0.02 \%$ for 12 seconds, immediately after excimer ablation. Then the eyes were irrigated by copious amounts of chilled balanced salt solution. At the end of the laser ablation, a high-oxygen-content silicone hydrogel soft contact lens was placed with instillation of a drop of topical steroid. The patients were treated with topical instillation of antibiotic drops; gatifloxacin $0.5 \%$ (Gatistar; Orchidia, Al-Obour, Egypt) six times daily for 1 week or till epithelial healing, topical instillation of steroid Prednisolone acetate drops (Orchapred, Orchidia) six times daily (tapered over 4 weeks and shifted to flourometholone $0.1 \%$ flucon (Alcon Laboratories, Macquarie Park, NSW, Australia) four times per day for another 1 month, and artificial tear drops six times daily for 3 months. An adjuvant therapy was added to the standard treatment regimen to augment the epithelial healing process in the form of vitamin C tablets $500 \mathrm{mg}$ twice per day and vitamin A tablets once per day both for 1 month. All patients were instructed to use a UV-blocker sunglass in sunlight for 1st month. At the first postoperative follow up, contact lens removal, or replacement (depending on the epithelial healing). Reepithelization and pain were noted.

\section{Postoperative assessment}

Postoperative assessment and follow ups were performed on day 1 and 3, at 1st week, and 1st, 3rd, 6th, 12th months. Both patients and the observer were masked to the assignment of the eyes. Postoperative corneal haze was observed at 1 week, 1, 3, 6 and 12 months and clinically graded with the slit-lamp biomicroscope according to the tabulation of the Fantes/Hanna scale [10]. Postoperative imaging with the Pentacam HR and Sirius device was done at 1, 3, 6 and 12 months follow ups. Other recorded parameters were delayed epithelial healing assessed with fluorescein staining, UCVA, manifest refraction, spherical equivalent, subjective pain at 1st and 3rd day assessed by patients scoring each eye on a scale of 5 points representing pain intensity from an Arabic translation of McGill Pain Questionnaire [11]. Contrast sensitivity was tested in mesopic conditions at 6 and 12 months by Frey chart panel CP-P600P (FreyMedical, Warsaw, Poland) using sinusoidal bar grading presented at different contrast level at 1.5, 3, 6, 12, 18 cycles per degree. Software then calculates the contrast sensitivity and figures it on the standard graph.

\section{Statistical analysis}

Data were analyzed by MedCalc Statistical Software ver. 18.9.1 (MedCalc Software, Ostend, Belgium). Data were described as mean \pm standard deviation and comparisons between groups were done by independent $t$-test where $p \leq$ 0.05 was set to be of significant level. Graphs were drawn by Minitab 18 Statistical Software (Minitab, State College, PA, USA).

AstigMATIC software 2018 (Mathworks, Natick, MA, USA) was used to calculate vector astigmatic correction of both groups. The software produces the four following standard graphs according to the standards of the Alpins method: (1) target-induced astigmatism (TIA) vector: this graph shows the range of astigmatism (magnitude and 
axis) that the surgery intended to induce. (2) Surgically-induced astigmatism (SIA) vector: this graph shows the range of achieved astigmatism cylinder and axis treatment and is used to compare the achieved (SIA) astigmatism treatment to the intended (TIA) treatment. (3) Difference vector: this graph shows remaining astigmatism and provides a summary of the astigmatic error considering both magnitude and axis. The difference vector is often used as an absolute measure of success and is preferably null. (4) Correction index: this graph shows the under/overcorrection of the astigmatism treatment. The correction index can also be used as a measure of success and is calculated as SIA divided by TIA. With an optimal surgical outcome it is equal to 1 , and is greater and smaller than 1 if an overcorrection and under-correction occurs, respectively. The blue regions, spreading from 0 to 30 degrees and from 150 to 180 degrees, highlight against-the-rule astigmatism, the red region, spreading from 60 to 120 degrees, highlights within-the-rule astigmatism, while unshaded white regions, spreading from 30 to 60 degrees and from 120 to 150 degrees, highlight oblique astigmatism [12].

\section{Results}

The study enrolled 58 eyes of 29 patients, then, two patients (four eyes) were excluded from the late follow-ups as mentioned before. Twenty-nine eyes (14 right eyes and 15 left eyes) enrolled in the TPRK group, and the contralateral eyes in the AAPRK group. There were seven males and 22 females, with an age of $31 \pm 6$ years (range, 20-45 years). Table 1 shows the preoperative baseline refraction and topographic data. There were no significant differenc-

Table 1. Preoperative data of AAPRK and TPRK

\begin{tabular}{|c|c|c|c|}
\hline Parameter & AAPRK (29 eyes) & TPRK (29 eyes) & $p$-value \\
\hline UCVA (Snellen's VA) & $0.27 \pm 0.15$ & $0.24 \pm 0.16$ & 0.4524 \\
\hline BCVA (Snellen's VA) & $0.96 \pm 0.16$ & $0.96 \pm 0.17$ & 0.8754 \\
\hline Sphere (D) & $-2.27 \pm 1.18$ & $-2.26 \pm 1.33$ & 0.9896 \\
\hline Cylinder (D) & $-1.00 \pm 0.69$ & $-1.12 \pm 0.77$ & 0.5332 \\
\hline Spherical equivalent (D) & $-2.77 \pm 1.2$ & $-2.86 \pm 1.2$ & 0.7697 \\
\hline K1 (D) & $42.7 \pm 1.58$ & $42.67 \pm 1.6$ & 1.0000 \\
\hline K2 (D) & $43.7 \pm 1.73$ & $43.78 \pm 1.81$ & 0.8572 \\
\hline $\mathrm{Km}(\mathrm{D})$ & $43.2 \pm 1.62$ & $43.20 \pm 1.68$ & 0.9358 \\
\hline Pachymetry apex $(\mu \mathrm{m})$ & $553 \pm 31$ & $553 \pm 32$ & 0.9560 \\
\hline Thinnest location $(\mu \mathrm{m})$ & $550 \pm 30$ & $550 \pm 32$ & 0.9728 \\
\hline Q value front & $-0.4 \pm 0.1$ & $-0.4 \pm 0.11$ & 0.8472 \\
\hline Ant $120 \mu \mathrm{m}$ Densito, total annulus (GSU) & $26.7 \pm 2.19$ & $26.79 \pm 2.38$ & 0.8754 \\
\hline Total cornea Densito, total annulus (GSU) & $18.58 \pm 1.3$ & $18.68 \pm 1.39$ & 0.7741 \\
\hline Total cornea RMS & $1.37 \pm 0.6$ & $1.39 \pm 0.53$ & 0.9423 \\
\hline HOA-RMS & $0.35 \pm 0.14$ & $0.34 \pm 0.06$ & 0.6796 \\
\hline LOA-RMS & $1.32 \pm 0.59$ & $1.34 \pm 0.54$ & 0.9116 \\
\hline Horizontal coma $(\mu \mathrm{m})$ & $-0.017 \pm 0.126$ & $0.009 \pm 0.144$ & 0.4633 \\
\hline Vertical coma $(\mu \mathrm{m})$ & $-0.062 \pm 0.19$ & $-0.059 \pm 0.149$ & 0.9410 \\
\hline Spherical aberrations $(\mu \mathrm{m})$ & $0.168 \pm 0.07$ & $0.164 \pm 0.082$ & 0.8293 \\
\hline Vertical trefoil $(\mu \mathrm{m})$ & $-0.0767 \pm 0.1231$ & $-0.0703 \pm 0.0812$ & 0.8194 \\
\hline Oblique trefoil $(\mu \mathrm{m})$ & $0.0036 \pm 0.0839$ & $0.0155 \pm 0.0914$ & 0.6172 \\
\hline
\end{tabular}

Values are presented as mean \pm standard deviation.

AAPRK = alcohol assisted photorefractive keratectomy; TPRK = transepithelial photorefractive keratectomy; UCVA = uncorrected visual acuity; $\mathrm{VA}=$ visual acuity; $\mathrm{BCVA}=$ best-corrected visual acuity; $\mathrm{D}=$ diopter; $\mathrm{K} 1$ = flat keratometry reading; $\mathrm{K} 2=$ steep keratometry reading; $\mathrm{Km}=$ mean keratometry; Densito = densitometry; GSU = greyscale unit; RMS = root mean square; HOA = higher order aberration; LOA $=$ lower order aberration. 
es in these baseline parameters between the two groups. The mean preoperative spherical equivalent of the patients was $-2.77 \pm 1.2$ and- $2.86 \pm 1.2 \mathrm{D}$ in AAPRK group and TPRK group, respectively, which was also not significantly different.

Both groups had large treatment zones with no statistically significant difference (AAPRK, $7.66 \pm 0.66 \mathrm{~mm}$ and TPRK, $7.7 \pm 0.68 \mathrm{~mm}$ with $p=0.7732$ ). The ablation depth was much deeper in the TPRK group $(103.13 \pm 25.5 \mu \mathrm{m})$ compared to AAPRK group $(60.9 \pm 28.7 \mu \mathrm{m})$ with a high statistically significant difference ( $p<0.0001)$, this is due to the $55 \mu \mathrm{m}$ epithelium removed by laser ablation.

\section{Postoperative epithelial healing and pain}

Epithelium healing was complete by the 3rd day in $62.1 \%$ of eyes in the alcohol assisted group and in $89.7 \%$ of eyes of the TPRK group. Postoperative pain was assessed at the 1st and 3rd postoperative days as mentioned in methodology and the results are shown in Table 2. First day postoperative pain was higher in TPRK group with statistically significant difference $(p=0.0134)$.

Table 2. First and 3rd day postoperative pain

\begin{tabular}{lrrr}
\hline Parameter & \multicolumn{1}{c}{ AAPRK } & \multicolumn{1}{c}{ TPRK } & $p$-value \\
\hline 1st day pain & $2 \pm 1.26$ & $2.9 \pm 1.11$ & $0.0134^{*}$ \\
3rd day pain & $0.71 \pm 1.23$ & $0.76 \pm 1.33$ & 0.9051 \\
\hline
\end{tabular}

Values are presented as mean \pm standard deviation.

AAPRK = alcohol assisted photorefractive keratectomy; TPRK = transepithelial photorefractive keratectomy.

"Statistically significant $(p \leq 0.05)$.

\section{Early postoperative visual results}

Table 3 demonstrates the results of the 3 rd day and 1st week postoperative follow-ups. None of the parameters compared showed a statistically significant difference between the two groups. By the 1st week postoperative, the UCVA was $0.81 \pm 0.24$ and $0.79 \pm 0.21$ for the alcohol assisted group and TPRK group, respectively, which has no statistically significant difference $(p=0.3719)$.

\section{Late postoperative results}

Table 4 shows the results of the 1st, 3rd, 6th, and 12th month follow-ups. The spherical error and the spherical equivalent were better in the TPRK at the 1st and 3rd months with statistically significant values. These differences narrowed by time, and were statistically insignificant at the 6th and 12th month follow-ups. However, this difference in spherical error and spherical equivalent did not affect the UCVA was at any point, as it showed similar levels at all follow-ups, to have a final acuity at 12 months of 1.47 \pm 0.39 and $1.57 \pm 0.38$ in the alcohol assisted group and TPRK group, respectively, with no statistically significant difference $(p=0.3719)$. Post-PRK haze showed progressive decrease over time in both groups with lower haze levels in the TPRK group, to reach a final level of $0.04 \pm 0.14$ and $0.02 \pm 0.1$ in the alcohol assisted group and TPRK group, respectively ( $p=0.5607)$.

Both lower and higher order aberrations showed continuous decrease over the 4 visits with a slightly more improvement in the TPRK group, to reach a level for lower order aberration-root mean square of $1.35 \pm 0.34$ and 1.21 $\pm 0.34(p=0.1851)$, and for higher order aberration-root

Table 3. Third day and 1st week follow-ups

\begin{tabular}{|c|c|c|c|c|c|c|}
\hline \multirow{2}{*}{ Parameter } & \multicolumn{3}{|c|}{ Third day postoperative } & \multicolumn{3}{|c|}{ First week postoperative } \\
\hline & AAPRK & TPRK & $p$-value & AAPRK & TPRK & $p$-value \\
\hline UCVA (Snellen's VA) & $0.66 \pm 0.21$ & $0.65 \pm 0.14$ & 0.9165 & $0.81 \pm 0.24$ & $0.79 \pm 0.21$ & 0.8163 \\
\hline Sphere (D) & $-0.13 \pm 1.63$ & $-0.34 \pm 0.95$ & 0.5571 & $-0.16 \pm 0.89$ & $-0.22 \pm 0.79$ & 0.7563 \\
\hline Cylinder (D) & $-0.91 \pm 0.65$ & $-0.91 \pm 0.78$ & 0.9964 & $-0.61 \pm 0.53$ & $-0.51 \pm 0.6$ & 0.4988 \\
\hline Spherical equivalent (D) & $-0.59 \pm 1.51$ & $-0.79 \pm 0.84$ & 0.5197 & $-0.46 \pm 0.95$ & $-0.48 \pm 0.92$ & 0.9401 \\
\hline Post PRK haze & $0.8 \pm 0.4$ & $0.9 \pm 0.4$ & 0.7562 & $0.81 \pm 0.44$ & $0.63 \pm 0.37$ & 0.1153 \\
\hline
\end{tabular}

Values are presented as mean \pm standard deviation.

AAPRK = alcohol assisted photorefractive keratectomy; TPRK = transepithelial photorefractive keratectomy; UCVA = uncorrected visual acuity; $\mathrm{VA}=$ visual acuity; $\mathrm{D}=$ diopter; $\mathrm{PRK}=$ photorefractive keratectomy. 
Table 4. Months 1, 3, 6, and 12 follow-ups results

\begin{tabular}{|c|c|c|c|c|c|c|c|c|c|}
\hline Parameter & Group & 1 mon Postop & $p$-value & 3 mon Postop & $p$-value & 6 mon Postop & $p$-value & 12 mon Postop & $p$-value \\
\hline \multirow{2}{*}{$\begin{array}{l}\text { UCVA } \\
\text { (Snellen's VA) }\end{array}$} & AAPRK & $1.03 \pm 0.24$ & 1.0000 & $1.12 \pm 0.28$ & 0.9367 & $1.36 \pm 0.41$ & 0.8361 & $1.47 \pm 0.39$ & 0.3719 \\
\hline & TPRK & $1.03 \pm 0.20$ & & $1.12 \pm 0.24$ & & $1.34 \pm 0.44$ & & $1.57 \pm 0.38$ & \\
\hline \multirow[t]{2}{*}{ Sphere (D) } & AAPRK & $-0.40 \pm 0.60$ & 0.0083 & $-0.37 \pm 0.52$ & 0.0395 & $-0.30 \pm 0.46$ & 0.3383 & $-0.45 \pm 0.52$ & 0.7107 \\
\hline & TPRK & $0.02 \pm 0.56$ & & $-0.09 \pm 0.41$ & & $-0.17 \pm 0.52$ & & $-0.39 \pm 0.46$ & \\
\hline \multirow[t]{2}{*}{ Cylinder (D) } & AAPRK & $-0.41 \pm 0.40$ & 0.7755 & $-0.45 \pm 0.34$ & 0.3355 & $-0.34 \pm 0.25$ & 0.6434 & $-0.24 \pm 0.27$ & 0.0695 \\
\hline & TPRK & $-0.44 \pm 0.29$ & & $-0.36 \pm 0.32$ & & $-0.38 \pm 0.33$ & & $-0.38 \pm 0.25$ & \\
\hline \multirow{2}{*}{$\begin{array}{l}\text { Spherical equivalent } \\
\text { (D) }\end{array}$} & AAPRK & $-0.61 \pm 0.63$ & 0.0177 & $-0.60 \pm 0.54$ & 0.0241 & $-0.47 \pm 0.46$ & 0.4220 & $-0.57 \pm 0.56$ & 0.9156 \\
\hline & TPRK & $-0.20 \pm 0.62$ & & $-0.27 \pm 0.44$ & & $-0.36 \pm 0.55$ & & $-0.58 \pm 0.47$ & \\
\hline \multirow[t]{2}{*}{ Post PRK haze } & AAPRK & $0.48 \pm 0.2$ & 0.1183 & $0.31 \pm 0.29$ & 0.3553 & $0.11 \pm 0.21$ & 0.1569 & $0.04 \pm 0.14$ & 0.5607 \\
\hline & TPRK & $0.38 \pm 0.2$ & & $0.23 \pm 0.23$ & & $0.03 \pm 0.12$ & & $0.02 \pm 0.1$ & \\
\hline \multirow[t]{2}{*}{ K1 (D) } & AAPRK & $40.56 \pm 2.12$ & 0.6344 & $40.70 \pm 2.12$ & 0.8041 & $41.09 \pm 1.62$ & 0.9243 & $40.78 \pm 1.82$ & 0.9419 \\
\hline & TPRK & $40.29 \pm 2.12$ & & $40.55 \pm 2.08$ & & $41.05 \pm 1.57$ & & $40.81 \pm 1.73$ & \\
\hline \multirow[t]{2}{*}{ K2 (D) } & AAPRK & $41.25 \pm 2.09$ & 0.5910 & $41.34 \pm 2.04$ & 0.7514 & $41.70 \pm 1.58$ & 0.8700 & $41.36 \pm 1.79$ & 0.9034 \\
\hline & TPRK & $40.94 \pm 2.21$ & & $41.16 \pm 2.14$ & & $41.63 \pm 1.63$ & & $41.43 \pm 1.76$ & \\
\hline \multirow[t]{2}{*}{$\mathrm{Km}$ (D) } & AAPRK & $40.89 \pm 2.09$ & 0.6209 & $41.01 \pm 2.07$ & 0.7924 & $41.39 \pm 1.60$ & 0.8964 & $41.08 \pm 1.80$ & 0.9482 \\
\hline & TPRK & $40.61 \pm 2.15$ & & $40.86 \pm 2.09$ & & $41.33 \pm 1.58$ & & $41.11 \pm 1.74$ & \\
\hline \multirow{2}{*}{$\begin{array}{l}\text { Pachymetry apex } \\
\qquad(\mu \mathrm{m})\end{array}$} & AAPRK & $502 \pm 38$ & 0.5828 & $509 \pm 40$ & 0.6681 & $511 \pm 39$ & 0.6664 & $508.67 \pm 35.92$ & 0.8674 \\
\hline & TPRK & $497 \pm 37$ & & $504 \pm 39$ & & $506 \pm 41$ & & $506.92 \pm 36.28$ & \\
\hline \multirow{2}{*}{$\begin{array}{l}\text { Thinnest location } \\
\qquad(\mu \mathrm{m})\end{array}$} & AAPRK & $500 \pm 37$ & 0.5662 & $505 \pm 39$ & 0.7108 & $509 \pm 39$ & 0.6506 & $506.54 \pm 35.59$ & 0.8172 \\
\hline & TPRK & $494 \pm 38$ & & $501 \pm 39$ & & $504 \pm 41$ & & $504.13 \pm 36.42$ & \\
\hline \multirow[t]{2}{*}{$\mathrm{Q}$ value front } & AAPRK & $0.03 \pm 0.29$ & 0.6432 & $0.00 \pm 0.26$ & 0.4941 & $0.01 \pm 0.23$ & 0.8177 & $0.02 \pm 0.23$ & 0.6978 \\
\hline & TPRK & $0.08 \pm 0.41$ & & $0.06 \pm 0.34$ & & $-0.01 \pm 0.29$ & & $-0.01 \pm 0.27$ & \\
\hline \multirow{2}{*}{$\begin{array}{l}\text { Ant } 120 \mu \mathrm{m} \text { Densito, } \\
\quad \text { total annulus (GSU) }\end{array}$} & AAPRK & $27.23 \pm 4.70$ & 0.1703 & $24.59 \pm 2.20$ & 0.3776 & $24.92 \pm 2.93$ & 0.3791 & $23.79 \pm 2.89$ & 0.8156 \\
\hline & TPRK & $25.80 \pm 2.68$ & & $24.05 \pm 2.09$ & & $24.25 \pm 2.49$ & & $23.62 \pm 2.23$ & \\
\hline \multirow{2}{*}{$\begin{array}{l}\text { Total cornea Densito, } \\
\text { total annulus (GSU) }\end{array}$} & AAPRK & $19.29 \pm 2.28$ & 0.2963 & $18.19 \pm 1.49$ & 0.1994 & $18.3 \pm 1.988$ & 0.4788 & $17.61 \pm 1.95$ & 0.7175 \\
\hline & TPRK & $18.71 \pm 1.74$ & & $17.67 \pm 1.31$ & & $18.00 \pm 1.87$ & & $17.43 \pm 1.51$ & \\
\hline \multirow[t]{2}{*}{ Total cornea RMS } & AAPRK & $1.74 \pm 0.72$ & 0.7594 & $1.51 \pm 0.40$ & 0.7666 & $1.44 \pm 0.46$ & 0.3995 & $1.47 \pm 0.33$ & 0.1495 \\
\hline & TPRK & $1.68 \pm 0.60$ & & $1.48 \pm 0.47$ & & $1.35 \pm 0.35$ & & $1.33 \pm 0.35$ & \\
\hline \multirow[t]{2}{*}{ HOA-RMS } & AAPRK & $0.64 \pm 0.16$ & 0.6709 & $0.59 \pm 0.14$ & 0.9421 & $0.57 \pm 0.11$ & 0.4238 & $0.58 \pm 0.08$ & 0.0935 \\
\hline & TPRK & $0.62 \pm 0.20$ & & $0.60 \pm 0.25$ & & $0.55 \pm 0.14$ & & $0.53 \pm 0.13$ & \\
\hline \multirow[t]{2}{*}{ LOA-RMS } & AAPRK & $1.60 \pm 0.74$ & 0.7717 & $1.38 \pm 0.42$ & 0.7209 & $1.31 \pm 0.50$ & 0.4830 & $1.35 \pm 0.34$ & 0.1851 \\
\hline & TPRK & $1.55 \pm 0.62$ & & $1.34 \pm 0.44$ & & $1.22 \pm 0.35$ & & $1.21 \pm 0.34$ & \\
\hline \multirow{2}{*}{$\begin{array}{l}\text { Horizontal coma } \\
\qquad(\mu \mathrm{m})\end{array}$} & AAPRK & $-0.01 \pm 0.17$ & 0.5566 & $-0.03 \pm 0.21$ & 0.2032 & $0.02 \pm 0.21$ & 0.9473 & $-0.02 \pm 0.21$ & 0.1724 \\
\hline & TPRK & $0.03 \pm 0.27$ & & $0.05 \pm 0.23$ & & $0.02 \pm 0.24$ & & $0.06 \pm 0.21$ & \\
\hline \multirow[t]{2}{*}{ Vertical coma $(\mu \mathrm{m})$} & AAPRK & $-0.25 \pm 0.23$ & 0.1009 & $-0.17 \pm 0.27$ & 0.5577 & $-0.11 \pm 0.26$ & 0.5391 & $-0.16 \pm 0.25$ & 0.3719 \\
\hline & TPRK & $-0.15 \pm 0.22$ & & $-0.12 \pm 0.29$ & & $-0.07 \pm 0.22$ & & $-0.10 \pm 0.20$ & \\
\hline \multirow{2}{*}{$\begin{array}{l}\text { Spherical aberrations } \\
\qquad(\mu \mathrm{m})\end{array}$} & AAPRK & $0.34 \pm 0.18$ & 0.7780 & $0.27 \pm 0.16$ & 0.7631 & $0.32 \pm 0.13$ & 0.1995 & $0.34 \pm 0.11$ & 0.1536 \\
\hline & TPRK & $0.32 \pm 0.23$ & & $0.29 \pm 0.21$ & & $0.27 \pm 0.17$ & & $0.29 \pm 0.14$ & \\
\hline \multirow[t]{2}{*}{ Vertical trefoil $(\mu \mathrm{m})$} & AAPRK & $-0.022 \pm 0.176$ & 0.4075 & $-0.053 \pm 0.148$ & 0.1577 & $-0.055 \pm 0.155$ & 0.3852 & $-0.08 \pm 0.11$ & 0.0961 \\
\hline & TPRK & $-0.061 \pm 0.179$ & & $-0.113 \pm 0.152$ & & $-0.093 \pm 0.161$ & & $-0.14 \pm 0.15$ & \\
\hline \multirow[t]{2}{*}{ Oblique trefoil $(\mu \mathrm{m})$} & AAPRK & $0.030 \pm 0.150$ & 0.1591 & $0.008 \pm 0.132$ & 0.1681 & $-0.016 \pm 0.118$ & 0.7342 & $0.00 \pm 0.11$ & 0.7150 \\
\hline & TPRK & $-0.020 \pm 0.107$ & & $-0.045 \pm 0.138$ & & $-0.028 \pm 0.131$ & & $-0.01 \pm 0.08$ & \\
\hline
\end{tabular}

Values are presented as mean \pm standard deviation.

Postop = postoperative; UCVA = uncorrected visual acuity; VA = visual acuity; AAPRK = alcohol assisted photorefractive keratectomy; $\mathrm{TPRK}$ = transepithelial photorefractive keratectomy; D = diopter; K1 = flat keratometry reading; K2 = steep keratometry reading; Km = mean keratometry; Densito = densitometry; GSU = greyscale unit; RMS = root mean square; HOA = higher order aberration; LOA = lower order aberration. 


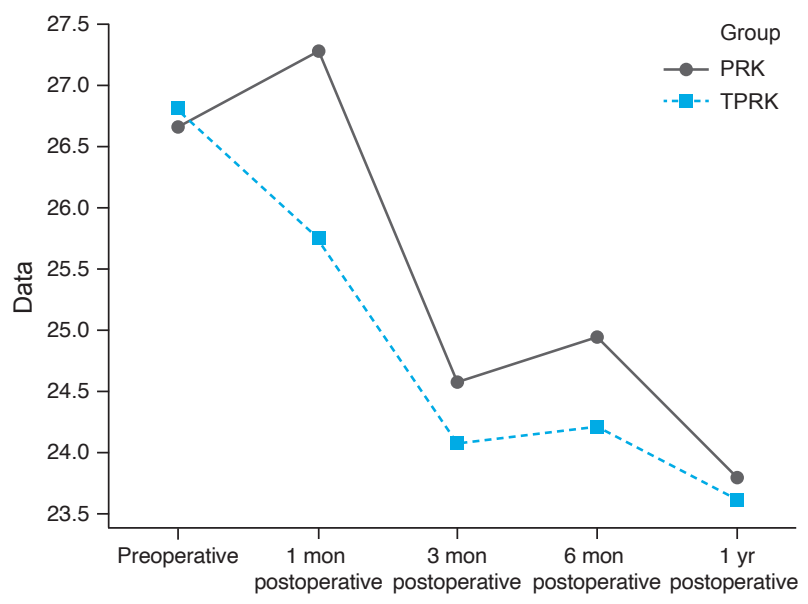

Fig. 1. Mean preoperative and postoperative average anterior 120 $\mu \mathrm{m}$ densitometry. PRK = photorefractive keratectomy; TPRK = transepithelial photorefractive keratectomy.

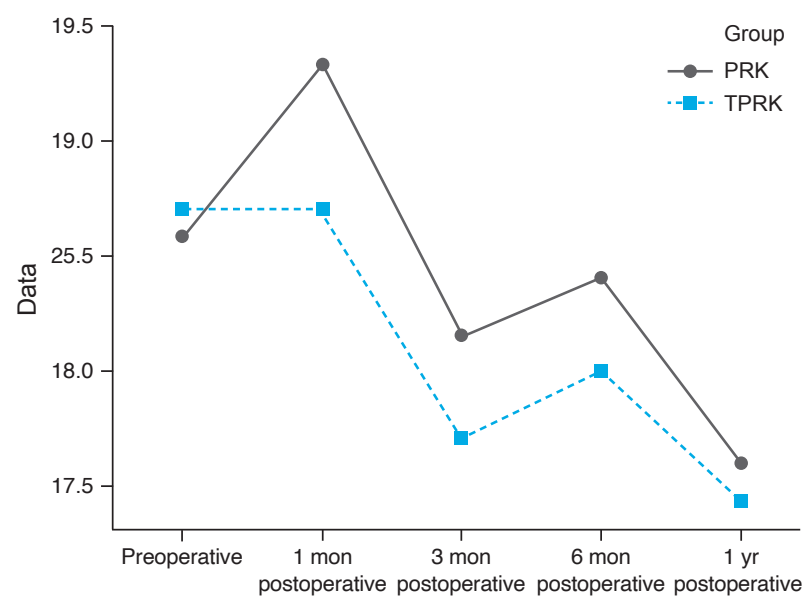

Fig. 2. Mean preoperative and postoperative average total corneal thickness densitometry. PRK = photorefractive keratectomy; TPRK $=$ transepithelial photorefractive keratectomy.

mean square $0.58 \pm 0.08$ and $0.53 \pm 0.13(p=0.0935)$ in the alcohol assisted group and TPRK group, respectively. Also, both levels did not show a significant difference between the two groups.

Densitometry levels were less in TPRK group and decreased by time, but the difference was not statistically significant (Fig. 1, 2).

Contrast sensitivity is a very important measure of visual function, especially in situations of low light, fog or glare, which are affected also by refractive laser correction. We measured contrast sensitivity at both low and high grating frequencies and found comparable contrast

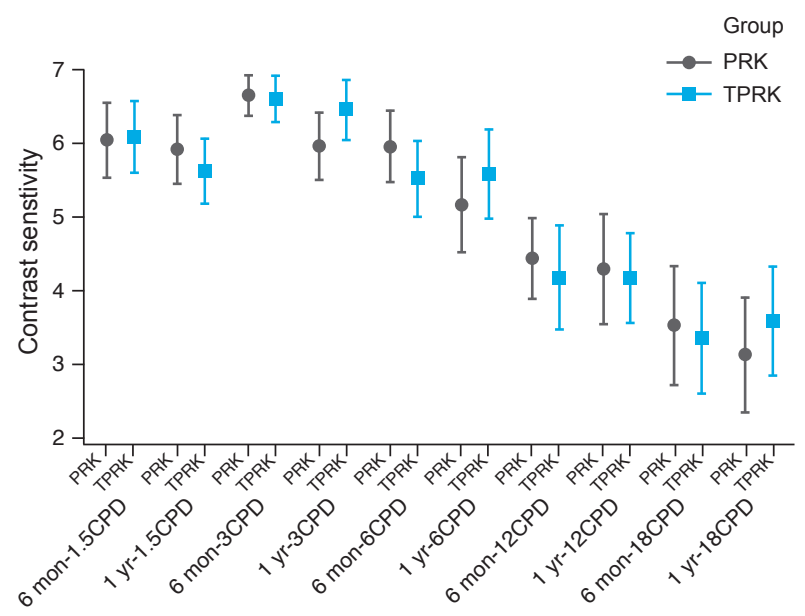

Fig. 3. Interval plot of contrast sensitivity at 6 months and 1 year. $95 \%$ confidence interval for the mean. PRK = photorefractive keratectomy; TPRK $=$ transepithelial photorefractive keratectomy; $\mathrm{CPD}=$ cycle per degree.

sensitivity curve for both AAPRK and TPRK groups at 6 months and 1 year (Fig. 3).

\section{Vector analysis of changes in refractive cylinder}

Astigmatism vector analysis was done at 1, 3, and 6 months and one year using the free online AstigMATIC software. Both techniques showed slight overcorrection of astigmatism at the 1st month analysis with a correction index of 1.22 and 1.20 for AAPRK and TPRK groups respectively. This overcorrection decreased by time to reach final values at one year of 0.99 and 1.05 for AAPRK and TPRK groups respectively (Fig. 4A, 4B, 5A, 5B, 6A, 6B).

Surgically induced astigmatic vector was within $0.5 \mathrm{D}$ of the intended target induced astigmatic vector. The difference vector (lower left graph in all figures) which is used as a measure of success as the graph shows the remaining astigmatism both in magnitude and axis- showed a mean difference of $0.45,0.44 \mathrm{D}$ in AAPRK and TPRK groups respectively at the 1st month. That decreased to reach 0.21 and 0.29 for same groups at 1st year.

\section{Discussion}

This study compares the outcomes of AAPRK and TPRK regarding safety and efficacy. Few reports comparing TPRK and AAPRK were published over the past 
A

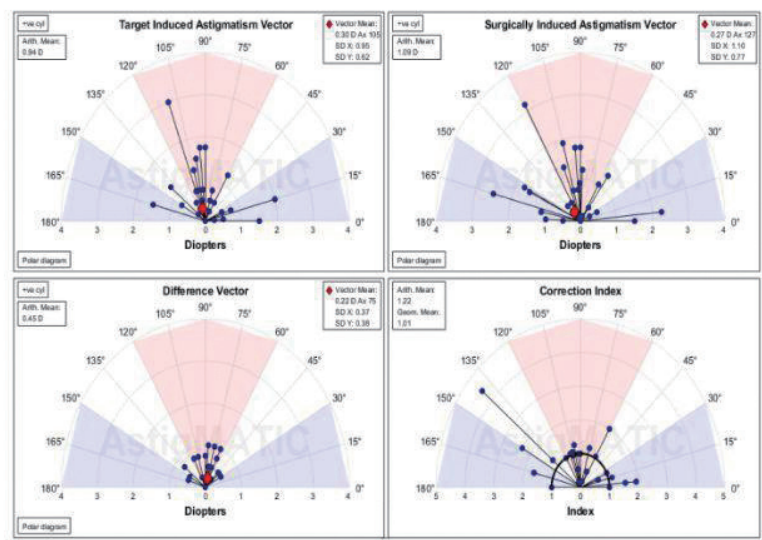

$\mathrm{B}$

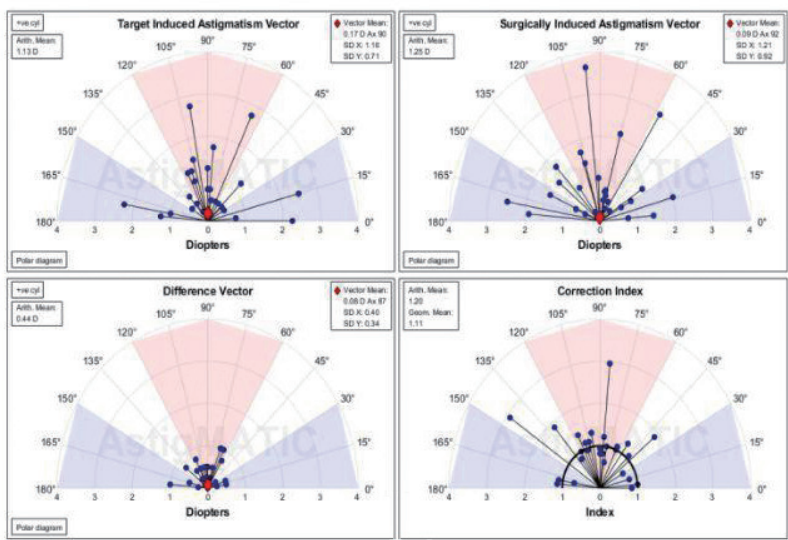

Fig. 4. Vector astigmatic analysis at 1 month. (A) Alcohol assisted photorefractive keratectomy and (B) transepithelial photorefractive keratectomy.

A

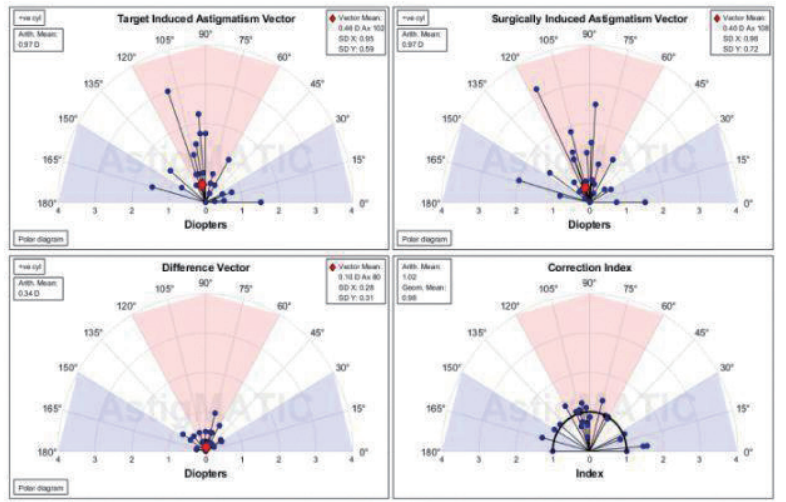

B

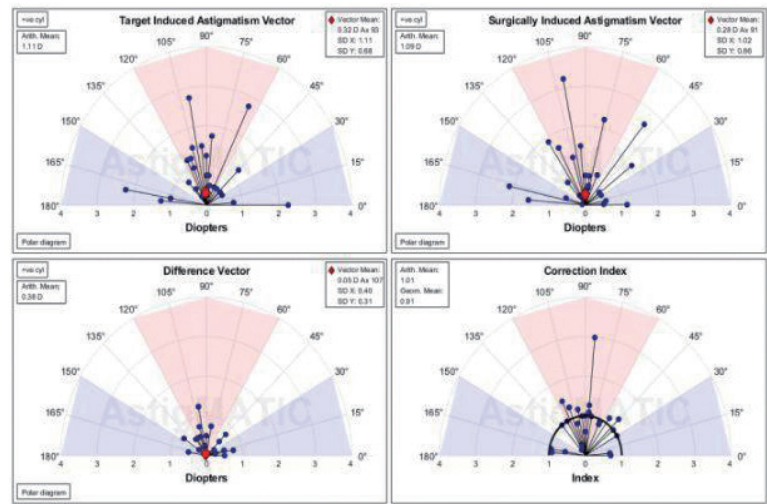

Fig. 5. Vector astigmatic analysis at 3 months. (A) Alcohol assisted photorefractive keratectomy and (B) transepithelial photorefractive keratectomy.

A

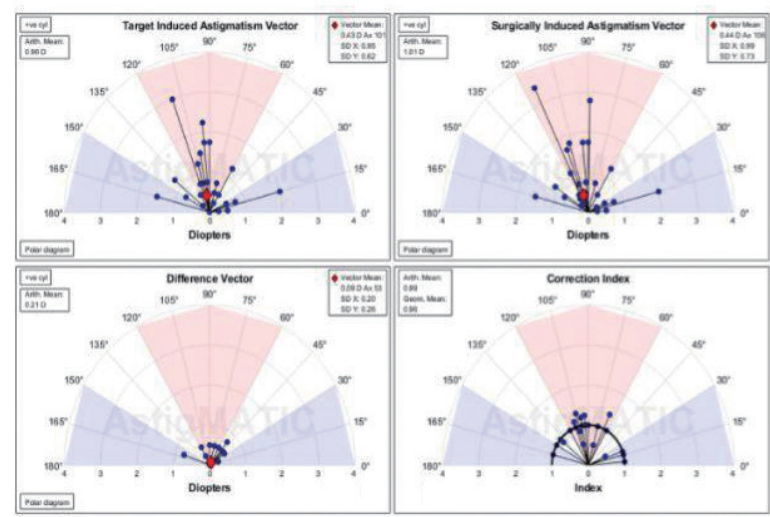

B

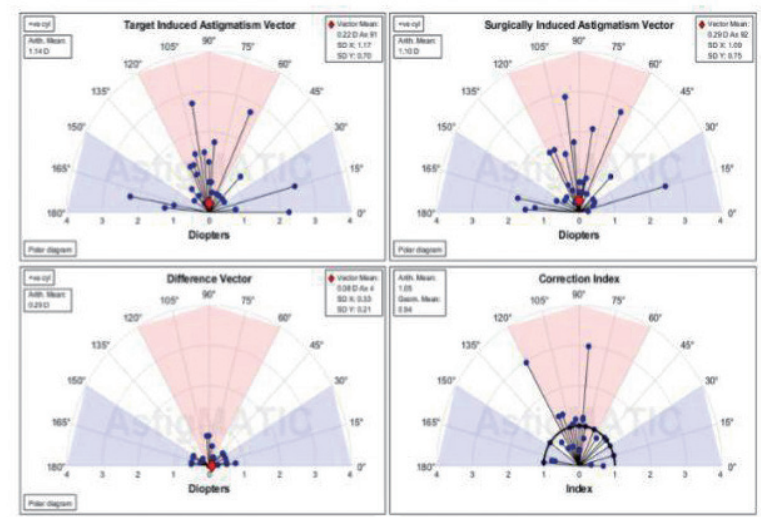

Fig. 6. Vector astigmatic analysis at 12 months. (A) Alcohol assisted photorefractive keratectomy and (B) transepithelial photorefractive keratectomy.

years. To our knowledge, there is only one published contralateral eye study comparing these two techniques [13]. Another contralateral eye study published by Zarei-Gha- navati et al. [14] was comparing TPRK and mechanical PRK.

There were no significant differences in the final postop- 
erative visual acuity, refractive error and higher order aberration in both groups, indicating that both techniques are equally effective. These results are consistent with the results of other studies. Ghobashy et al. [15] had an UCVA at 6 months of $1.1 \pm 0.32$ and $1.1 \pm 0.11$ in TPRK and AAPRK respectively with a $p$-value of 0.268 . Kaluzny et al. [16] found that the mean postoperative higher order aberrations RMS for $4 \mathrm{~mm}$ pupil was $0.21 \pm 0.23$ in the TPRK group and $0.19 \pm 0.12$ in the AAPRK group ( $p=0.37)$. In the study conducted by Bakhsh et al. [13] the mean postoperative manifest refractive spherical equivalent at 6 months was $-0.0500 \pm 0.337 \mathrm{D}$ in the TPRK group and $-0.0450 \pm$ $0.338 \mathrm{D}$ in the AAPRK group $(p=1)$ [13]. Moreover, both treatment modalities resulted in a very comparative contrast senstivity graphs for both low and high grating frequencies. This leads to comparable visual functions in both low and high contrast situations.

The most important parameters that showed significant differences between the two groups were the 3rd day postoperative epithelium healing and first postoperative day pain. The TPRK had a higher percentage of healing $(89.7 \%$ of the eyes) denoting that it has a slightly higher safety profile. This is consistent with the results of Bakhsh et al. who found that complete corneal epithelial healing time had a shorter mean in the TPRK group than in AAPRK, in which it was $3.20 \pm 0.686$ and $4.60 \pm 1.969$ days, respectively $(p<0.001)$ [13]. Zarei-Ghanavati et al. [14] also found shorter re-epithelialization time in the TPRK group than in mechanical PRK group of $3.28 \pm 0.60$ and $5.10 \pm$ 0.81 days, respectively with a $p$-value of 0.000 . This also consistent with the results of Lee et al. [17] who compared the epithelial healing in the different epithelial removal techniques in PRK and found that TPRK had the fastest healing. This faster healing may be attributed to the fact that the area of epithelial shed in TPRK, being done by laser, is limited to ablation zone only not as what happen with alcohol or mechanical removal where a bigger area is removed and possible epitheliotoxic effect of alcohol.

Despite that, to our surprise, the TPRK eyes had higher first postoperative pain levels compared to AAPRK group. Kaluzny et al. [16] found that there was no difference in pain intensity during 1st day after the surgery $(p=0.86)$. This is contradicting with the results of Fadlallah et al. [5], in which their postoperative pain score at 48 hours was 2.0 in the TPRK and 4.5 in the AAPRK $(p=0.02)$. It is also contradicting with the results of Ghobashy et al. [15] who found pain score at first day of $2.5 \pm 1.3$ in TPRK group and $3 \pm 1.0$ in AAPRK group but the difference was statistically not significant ( $p=0.292$ ). They reassessed pain score at 1 st week and it was 0 for TPRK and $0.75 \pm 0.96$ for AAPRK but again the difference was statistically not significant $(p=0.084)$. This contradiction in their results regarding first day pain may be attributed to the fact that they found there was a strong positive correlation $(+0.904)$ between wider optical zone and postoperative pain in AAPRK (who had larger optical zones than TPRK group). The difference in the 1st week could be attributed to the faster healing in TPRK group. Another factor that we might put in consideration is that in both studies the patients were not blinded to the technique they underwent which might put them under the psychological illusion that using laser to remove epithelium should not be that painful. Since our study was a contralateral eye study, we think that this gives the patient the chance to compare between the pain both eyes and this might support that TPRK is more painful in the first postoperative day. The contralateral eye study by Zarei-Ghanavati et al. [14] comparing TPRK and mechanical PRK supported our results. They found that pain score at day 1 was $5.12 \pm 2.49$ and $2.52 \pm$ 2.21 for TPRK group and mechanical PRK group, respectively with a statistically significant difference $(p=0.000)$. Their pain score at day 3 was $3.08 \pm 2.36$ and $1.78 \pm 03$ for TPRK group and mechanical PRK group, respectively which was also statistically significant $(p=0.010)$ [14] Also our results are consistent with Kanitkar et al. [18], in which their pain score was less in AAPRK than in the TPRK group. The higher postoperative pain in the TPRK group is most probably attributed to the higher ablation depth that uses more laser energy, and leads to the release of higher amounts of cytokines. Liu et al. [19] found in their study on rabbit eyes, a direct relation between the total of laser energy delivered to the eye and the amount of cytokine released.

This difference in pain between the two groups is important information to be added to the counseling of patients undergoing PRK and to be put in mind of the surgeons when choosing the suitable technique for their patients.

The densitometry levels were correlated with postoperative haze that was less in TPRK group and decreased by time in both groups. We could not find previous studies that did densitometry comparison between different PRK 
modalities, but some papers compared postoperative haze. Bakhsh et al. [13] assessed postoperative corneal haze using Fantes score. They found a lower incidence of postoperative corneal haze for the TPRK group compared to the AAPRK group at all tested time points: 1 week, 1 month, and 3 months, in which the mean results were $0.300 \pm$ $0.432,0.150 \pm 0.280$, and $0.100 \pm 0.236$ in the TPRK group, respectively, and the corresponding values in the AAPRK group were $0.530 \pm 0.876,0.350 \pm 0.687$, and $0.200 \pm 0.402$ with statistically significant differences in all tested time points, where the $\mathrm{P}$ values were $0.019,0.008$, and 0.033 , respectively. They also found that during the follow-up the corneal haze intensity had a tendency to decrease until reaching postoperative 6 months, where there was no haze in both the groups. This difference in corneal haze may be related to the findings of Helena et al. [20] study, in which they reported quantitative and qualitative differences in keratocyte apoptosis among LASIK, epithelial scrape-PRK and TPRK. They performed the different techniques on rabbits' eyes and found that TPRK was associated with the lowest levels of central corneal apoptosis, even if the stromal surface was scrapped after the procedure. Keratocyte apoptosis was confined to the superficial stroma extending to a depth of approximately 50 to $75 \mu \mathrm{m}$ after epithelial scrape-PRK and TPRK.

Astigmatic vector analysis showed that both groups achieved a near perfect correction of astigmatism with a difference between target and achieved astigmatic correction of less than $0.5 \mathrm{D}$ in the 1st month, and around $0.25 \mathrm{D}$ by the 1st year and, a correction index at 6 months of 1.02 and 1.01 and continued at one year to reach a value of 0.99 and 1.05 for AAPRK and TPRK groups respectively. That maeans precise astigmatic correction with no over or under correction than intended target. This indicates that both techniques are effective, safe and with high stability regarding astigmatic correction.

There are some limitations to this study such as we had two patients who were excluded from the long-term follow-up. The importance of this study is that it is a contralateral eye study comparing TPRK and AAPRK with masking for the patient with the technique used in each eye. The study compared the two techniques from different aspects regarding efficacy and safety. Also, to our knowledge, this is the first study that compares the two techniques regarding densitometry and contrast sensitivity.

\section{Conflict of Interest}

No potential conflict of interest relevant to this article was reported.

\section{References}

1. Munnerlyn CR, Koons SJ, Marshall J. Photorefractive keratectomy: a technique for laser refractive surgery. $J$ Cataract Refract Surg 1988;14:46-52.

2. Ghoreishi M, Attarzadeh H, Tavakoli M, et al. Alcohol-assisted versus mechanical epithelium removal in photorefractive keratectomy. J Ophthalmic Vis Res 2010;5:223-7.

3. Clinch TE, Moshirfar M, Weis JR, et al. Comparison of mechanical and transepithelial debridement during photorefractive keratectomy. Ophthalmology 1999;106:483-9.

4. Buzzonetti L, Petrocelli G, Laborante A, et al. A new transepithelial phototherapeutic keratectomy mode using the NIDEK CXIII excimer laser. J Refract Surg 2009;25(1 Suppl):S122-4.

5. Fadlallah A, Fahed D, Khalil K, et al. Transepithelial photorefractive keratectomy: clinical results. J Cataract Refract Surg 2011;37:1852-7.

6. Luger MH, Ewering T, Arba-Mosquera S. Consecutive myopia correction with transepithelial versus alcohol-assisted photorefractive keratectomy in contralateral eyes: one-year results. J Cataract Refract Surg 2012;38:1414-23.

7. Lin DT, Holland SP, Verma S, et al. Immediate and short term visual recovery after SmartSurf(ACE) photorefractive keratectomy. J Optom 2019;12:240-7.

8. Vinciguerra P, Camesasca FI, Vinciguerra R, et al. Advanced surface ablation with a new software for the reduction of ablation irregularities. J Refract Surg 2017;33:89-95.

9. Aslanides IM, Padroni S, Arba Mosquera S, et al. Comparison of single-step reverse transepithelial all-surface laser ablation (ASLA) to alcohol-assisted photorefractive keratectomy. Clin Ophthalmol 2012;6:973-80.

10. Fantes FE, Hanna KD, Waring GO 3rd, et al. Wound healing after excimer laser keratomileusis (photorefractive keratectomy) in monkeys. Arch Ophthalmol 1990;108:665-75.

11. Melzack R. The McGill Pain Questionnaire: major properties and scoring methods. Pain 1975;1:277-99.

12. Gauvin M, Wallerstein A. AstigMATIC: an automatic tool for standard astigmatism vector analysis. BMC Ophthalmol 2018;18:255. 
13. Bakhsh AM, Elwan SAM, Chaudhry AA, et al. Comparison between transepithelial photorefractive keratectomy versus alcohol-assisted photorefractive keratectomy in correction of myopia and myopic astigmatism. J Ophthalmol 2018;2018:5376235.

14. Zarei-Ghanavati S, Shandiz JH, Abrishami M, Karimpour M. Comparison of mechanical debridement and trans-epithelial myopic photorefractive keratectomy: a contralateral eye study. J Curr Ophthalmol 2019;31:135-41.

15. Ghobashy WA, Shahin ME, Kolkailah KA. Transepithelial photorefractive keratectomy versus conventional alcohol-assisted photorefractive keratectomy for correction of mild and moderate myopia. J Egypt Ophthalmol Soc 2014;107:1-14.

16. Kaluzny BJ, Cieslinska I, Mosquera SA, Verma S. Single-step transepithelial PRK vs alcohol-assisted PRK in myopia and compound myopic astigmatism correction.
Medicine (Baltimore) 2016;95:e1993.

17. Lee HK, Lee KS, Kim JK, et al. Epithelial healing and clinical outcomes in excimer laser photorefractive surgery following three epithelial removal techniques: mechanical, alcohol, and excimer laser. Am J Ophthalmol 2005;139:5663.

18. Kanitkar KD, Camp J, Humble H, et al. Pain after epithelial removal by ethanol-assisted mechanical versus transepithelial excimer laser debridement. J Refract Surg 2000;16:519-22.

19. Liu L, Cheng W, Wu D, et al. The differential expression of cytokines and growth factors after SMILE compared with FS-LASIK in rabbits. Invest Ophthalmol Vis Sci 2020;61:55.

20. Helena MC, Baerveldt F, Kim WJ, Wilson SE. Keratocyte apoptosis after corneal surgery. Invest Ophthalmol Vis Sci 1998:39:276-83. 